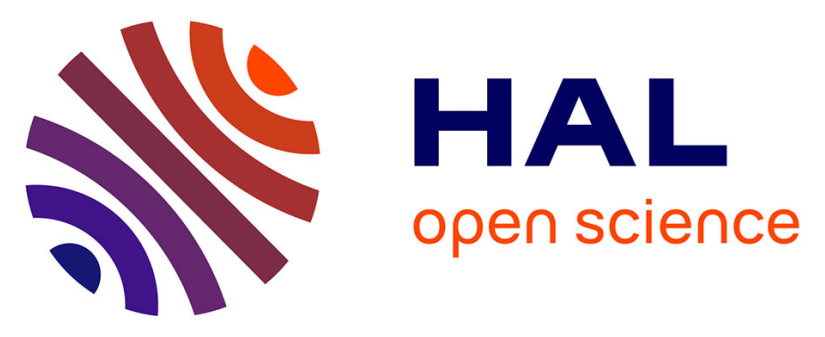

\title{
Molecular profile and proangiogenic activity of the adipose-derived stromal vascular fraction used as an autologous innovative medicinal product in patients with systemic sclerosis
}

Jeremy Magalon, Melanie Velier, Stephanie Simoncini, Pauline Francois, Baptiste Bertrand, Aurélie Daumas, Audrey Benyamine, Romain Boissier, Laurent Arnaud, Luc Lyonnet, et al.

\section{- To cite this version:}

Jeremy Magalon, Melanie Velier, Stephanie Simoncini, Pauline Francois, Baptiste Bertrand, et al.. Molecular profile and proangiogenic activity of the adipose-derived stromal vascular fraction used as an autologous innovative medicinal product in patients with systemic sclerosis. Annals of the Rheumatic Diseases, 2019, 78 (3), pp.391-398. 10.1136/annrheumdis-2018-214218 . hal-02555747

\section{HAL Id: hal-02555747 \\ https://hal-amu.archives-ouvertes.fr/hal-02555747}

Submitted on 27 Apr 2020

HAL is a multi-disciplinary open access archive for the deposit and dissemination of scientific research documents, whether they are published or not. The documents may come from teaching and research institutions in France or abroad, or from public or private research centers.
L'archive ouverte pluridisciplinaire HAL, est destinée au dépôt et à la diffusion de documents scientifiques de niveau recherche, publiés ou non, émanant des établissements d'enseignement et de recherche français ou étrangers, des laboratoires publics ou privés.

\section{(ㅇ)(1) $\$$}

Distributed under a Creative Commons Attribution - NonCommerciall 4.0 International 


\title{
Molecular profile and proangiogenic activity of the adipose-derived stromal vascular fraction used as an autologous innovative medicinal product in patients with systemic sclerosis
}

\author{
Jérémy Magalon, 1,2 Mélanie Velier, ${ }^{1,2}$ Stéphanie Simoncini, ${ }^{2}$ Pauline François, ${ }^{1,2}$ \\ Baptiste Bertrand, ${ }^{2,3}$ Aurélie Daumas, ${ }^{4}$ Audrey Benyamine, ${ }^{2,4}$ Romain Boissier, ${ }^{2,5}$ \\ Laurent Arnaud, ${ }^{6}$ Luc Lyonnet, ${ }^{6}$ Samantha Fernandez, ${ }^{7}$ Françoise Dignat-George, ${ }^{2,6}$ \\ Dominique Casanova, ${ }^{3}$ Benjamin Guillet, ${ }^{2,7}$ Brigitte Granel, ${ }^{2,4}$ Pascale Paul, ${ }^{1,2}$ \\ Florence Sabatier ${ }^{1,2}$
}

Handling editor Josef S Smolen

- Additional material is published online only. To view please visit the journal online (http://dx.doi.org/10.1136/ annrheumdis-2018-214218).

For numbered affiliations see end of article.

\section{Correspondence to} Professor Florence Sabatier, Cell Therapy Department, Hôpital de la Conception, Assistance Publique Hôpitaux de Marseille, Marseille 13005, France; florence.sabatier@ap-hm.fr

Received 31 July 2018 Revised 31 October 2018 Accepted 16 November 2018

Check for updates

(C) Author(s) (or their employer(s)) 2019. No commercial re-use. See rights and permissions. Published by BMJ.

\section{To cite: Magalon J,}

Velier M, Simoncini S, et al. Ann Rheum Dis Epub ahead of print: [please include Day Month Year]. doi:10.1136/ annrheumdis-2018-214218

\section{ABSTRACT}

Objective The autologous stromal vascular fraction (SVF) from adipose tissue is an alternative to cultured adipose-derived stem cells for use in regenerative medicine and represents a promising therapy for vasculopathy and hand disability in systemic sclerosis (SSc). However, the bioactivity of autologous SVF is not documented in this disease context. This study aimed to compare the molecular and functional profiles of the SVF-based medicinal product obtained from SSc and healthy subjects.

Methods Good manufacturing practice (GMP)-grade SVF from 24 patients with SSC and 12 healthy donors (HD) was analysed by flow cytometry to compare the distribution of the CD45- and CD45+ haematopoietic cell subsets. The ability of SVF to form a vascular network was assessed using Matrigel in vivo assay. The transcriptomic and secretory profiles of the SSC-SVF were assessed by RNA sequencing and multiplex analysis, respectively, and were compared with the HD-SVF. Results The distribution of the leucocyte, endothelial, stromal, pericyte and transitional cell subsets was similar for SSC-SVF and HD-SVF. SSc-SVF retained its vasculogenic capacity, but the density of neovessels formed in SVF-loaded Matrigel implanted in nude mice was slightly decreased compared with HD-SVF. SSc-SVF displayed a differential molecular signature reflecting deregulation of angiogenesis, endothelial activation and fibrosis.

Conclusions Our study provides the first evidence that SSc does not compromise the vascular repair capacity of SVF, supporting its use as an innovative autologous biotherapy. The characterisation of the specific SScSVF molecular profile provides new perspectives for delineating markers of the potency of SVF and its targets for the treatment of SSC.

\section{INTRODUCTION}

Systemic sclerosis (SSc) is a rare systemic autoimmune disease characterised by inflammation, fibrosis and vasculopathy. ${ }^{12}$ The key clinical manifestations of SSc are skin thickening and tightening and Raynaud's phenomenon. These symptoms typically coexist with gastrointestinal, cardiopulmonary

\section{Key messages}

What is already known about this subject?

- Autologous adipose tissue-derived stromal vascular fraction (SVF) represents a promising therapy for ischaemic vasculopathy and hand disability in systemic sclerosis (SSc), but the impact of the disease on the bioactivity of SVF has never been investigated.

What does this study add?

- Our study provides the first characterisation of GMP-grade autologous SVF used as an experimental advanced therapy medicinal products (ATMP) in patients with SSC.

- SVF from SSc presents similar distribution of haematopoietic and regenerative subpopulations compared with healthy donors' SVF.

- SSc context does not compromise the vasculogenic capacity of SVF in vitro and in vivo.

- SSc-SVF presents a molecular signature mainly affecting endothelial and stromal cells and reflecting deregulation of angiogenesis, endothelial activation and fibrosis.

How might this impact on clinical practice or future developments?

- This study supports the rationale for autologous SSc-SVF therapeutic use and delineates new perspectives and targets for potential cell engineering strategies to optimise SVF-based therapeutic approaches in a personalised way.

and renal involvement, leading to high morbidity. ${ }^{3-5}$ The diffuse cutaneous form of the disease is associated with a worse outcome compared with limited cutaneous SSc. ${ }^{67}$

The injection of autologous stem/progenitor cells is emerging as a therapeutic option in SSc. Haematopoietic stem cell transplantation can achieve long-term benefits but display high toxicity. ${ }^{8} 9$ Adipose-derived stromal/stem cells (ASC) can also 
beneficially impact dysimmunity, vascular dysfunction and extracellular matrix production. ASCs are multipotent cells, obtained after cell culture, able to exert paracrine proangiogenic and immunomodulatory effects through the secretion of various growth factors and cytokines. ${ }^{10-12}$ ASC has been shown to reduce fibrosis in a murine model of diffuse SSc through normalisation of extracellular matrix remodelling and inflammation and stimulation of antioxidant defences. ${ }^{13}$ Translation of the use of ASC to the clinic was performed with a favourable safety profile and resulted in significant skin improvement in six patients with localised scleroderma. ${ }^{14}$

To circumvent the issues with ex vivo cell expansion, the use of the autologous stromal vascular fraction (SVF), obtained through enzymatic digestion of adipose tissue, is becoming an alternative to ASC in regenerative medicine, particularly in the context of vasculopathy and fibrosis. ${ }^{15}$ SVF is a dynamic and heterogeneous cell population including mesenchymal-like stem/stromal cells (MSC), endothelial progenitor cells (EPC), pericytes, haematopoietic and immune cells that recapitulates the variety of cells present in the adipose tissue vasculature and allows the onset of synergistic mechanisms promoting vascular repair. ${ }^{16-18}$ We recently reported the results from a phase I clinical trial showing the safety of local injection of autologous SVF cells in patients with SSc with hand disability. ${ }^{19}$ Changes in secondary endpoints indicated potential efficacy in the improvement of Raynaud's phenomenon, digital ulcers, hand pain and global quality of life at 6 and 24 months compared with baseline. ${ }^{19} 20$ The predominant improvement of peripheral vascular manifestations was consistent with the beneficial proangiogenic effects of SVF reported in experimental models and emerging clinical trials addressing ischaemic diseases. ${ }^{21-23}$ However, alterations of the progenitor cell-dependent endogenous capacity for vascular repair are involved in the SSc pathogenesis, ${ }^{24}{ }^{25}$ which raises questions about the therapeutic potency of autologous SVF in this disease. Conflicting results have arisen from studies comparing MSC from patients with SSc and healthy donors (HD). Bone marrow-derived MSC (BM-MSC) from patients with SSc displays enhanced TGF- $\beta$ receptor II expression and $1 \alpha 2$ collagen synthesis. ${ }^{26}$ This myofibroblast-like phenotype indicates that the SSc microenvironment may limit the therapeutic use of autologous MSC. The coculture of SSc-BM-MSC with HD microvascular endothelial cells reprogrammes these cells towards a proangiogenic phenotype. ${ }^{27}$ In contrast, the phenotypic and functional properties of ASC were recently reported to be unaffected in patients with SSc. ${ }^{28}$ In addition, blood-derived endothelial colony-forming cells from patients with SSc show an altered gene expression profile that may compromise their vascular repair capacity. ${ }^{29} 30$ However, no study has yet investigated whether SSc impairs the bioactivity of adipose tissue-derived SVF.

The aim of this study was to provide extensive characterisation of therapeutic-grade-SVF from patients with SSc (SSc-SVF) in comparison with HD-SVF. The phenotypic, transcriptomic and angiogenic properties of both fractions were assessed to refine the appraisal of autologous SVF-based therapeutic options in SSc.

\section{MATERIALS AND METHODS \\ Donor specifications}

SVF from patients with SSc was obtained from the cell biobank ( ${ }^{\circ} \mathrm{CD}-2011-1331$ attached to the Scleradec II clinical trial : ClinicalTrials.gov NCT02558543). SVF from HD was obtained from surgical residues of adipose tissue after liposuction for
Table 1 Baseline characteristics of patients with SSC and HD

\begin{tabular}{|c|c|c|c|}
\hline & SSc, $n=24$ & $H D, n=12$ & $P$ value \\
\hline Gender, female/male & $23 / 1$ & $10 / 2$ & - \\
\hline Age, years, mean \pm SEM & $57.4 \pm 2.5$ & $43 \pm 2.7$ & $0.0013^{*}$ \\
\hline Body mass index $\left(\mathrm{kg} / \mathrm{m}^{2}\right.$, mean $\left.\pm \mathrm{SEM}\right)$ & $24.1 \pm 0.74$ & $24.4 \pm 0.7$ & 0.772 \\
\hline CRP (median, 25-75th percentile) & $2.2(1.1-6.2)$ & $1.7(1-2.4)$ & 0.734 \\
\hline $\begin{array}{l}\text { Estimated glomerular filtration rate }(\mathrm{mL} / \\
\left.\mathrm{min} / 1.73 \mathrm{~m}^{2}\right)\end{array}$ & $92 \pm 3.6$ & $102 \pm 8.3$ & 0.204 \\
\hline \multicolumn{4}{|l|}{ Cardiovascular risk factors (\%) } \\
\hline Tobacco & 12.5 & 8 & \\
\hline Diabetes & 0 & 0 & - \\
\hline Arterial hypertension & 0 & 0 & - \\
\hline Hypercholesterolaemia & 8 & 0 & 0.347 \\
\hline \multicolumn{4}{|l|}{ Disease characteristics } \\
\hline Disease duration from diagnosis (years) & $6.7 \pm 0.9$ & - & - \\
\hline Early SSC disease $<4$ years & $3(12.5 \%)$ & - & - \\
\hline History of Raynaud's phenomenon (years) & $10(4.2-14.5)$ & - & - \\
\hline $\begin{array}{l}\text { SSc subclassification: diffuse vs limited } \\
(\%)\end{array}$ & $\begin{array}{l}9(37.5) \text { vs } 15 \\
(62.5)\end{array}$ & - & - \\
\hline Medsger's Severity Scale & $2(2-3)$ & - & - \\
\hline Total Modified Rodnan Skin Score & $\begin{array}{l}10(6.25- \\
16.5)\end{array}$ & - & - \\
\hline Digital ulcers (active) & $29 \%$ & - & - \\
\hline Cochin Hand Function Score & $39.8 \pm 1.8$ & - & - \\
\hline SHAQ score & $1.3 \pm 0.07$ & - & - \\
\hline Raynaud's Condition Score & $5.2 \pm 0.5$ & - & - \\
\hline Total lung capacity (\% of predicted value) & $93 \pm 3$ & - & - \\
\hline $\begin{array}{l}\text { Forced vital capacity (\% of predicted } \\
\text { value) }\end{array}$ & $99 \pm 4$ & - & - \\
\hline
\end{tabular}

Alveolar capillary diffusing capacity (\% of $55 \pm 3$

predicted value)

Autoantibodies (\% positive)

\begin{tabular}{llll} 
Antinuclear antibodies & 100 & - & - \\
\hline Antitopoisomerase-1 antibodies (Scl70) & 30.4 & - & - \\
\hline Anticentromere antibodies & 27.3 & - & - \\
\hline Anti-RNA polymerase III antibodies & 30 & - & - \\
Systemic sclerosis medications, n, \% & & - & - \\
$\begin{array}{l}\text { Calcium-channel blockers, ongoing } \\
\text { (previous) }\end{array}$ & $9,37.5(10$, & - & - \\
Bosentan, ongoing (previous) & $41.7)$ & & \\
& $6,25.0(4$, & - & - \\
Prednisone <10 mg/day, ongoing & $16.7)$ & & \\
(previous) & $8,33.3(1$, & - & - \\
Methotrexate, ongoing (previous) & $4.2)$ & $5,20.8(1,4.2)$ & - \\
Iloprost infusion, ongoing (previous) & $0,0(11,45.8)$ & - \\
\hline
\end{tabular}

Data are mean \pm SEM or median (25-75th percentile range) or $n(\%)$ of patients.

- , Not applicable in HD.

${ }^{*} \mathrm{P}<0.05$.

$\mathrm{CRP}, \mathrm{C}$ reactive protein; $\mathrm{HD}$, healthy donor; $\mathrm{SHAQ}$, scleroderma health assessment questionnaire; SSc, systemic sclerosis.

cosmetic purposes. The materials and methods are described in the online supplementary text.

\section{RESULTS}

\section{Characteristics of the patients}

The therapeutic-grade SVFs from 24 patients with SSc (15 limited and 9 diffuse cutaneous forms) were analysed in comparison with the SVF from 12 HDs. The demographic and standard biochemical data of the patients and control groups were similar except in age (table 1$)$. 
A

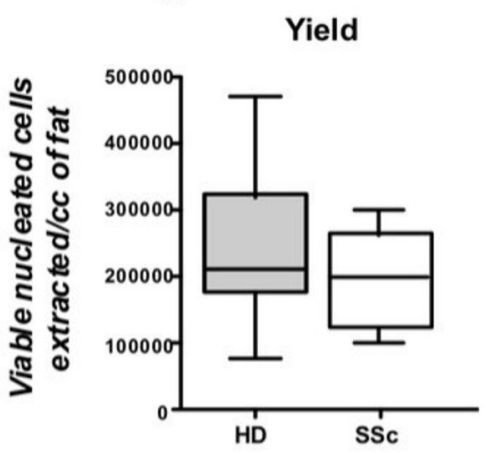

B

Viability

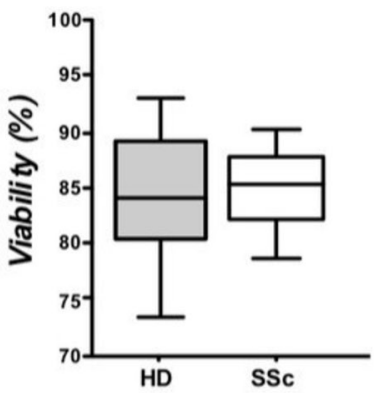

C

Clonogenic activity

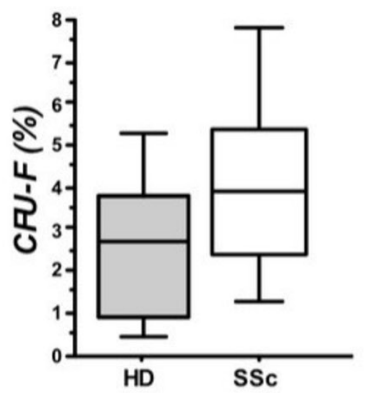

D

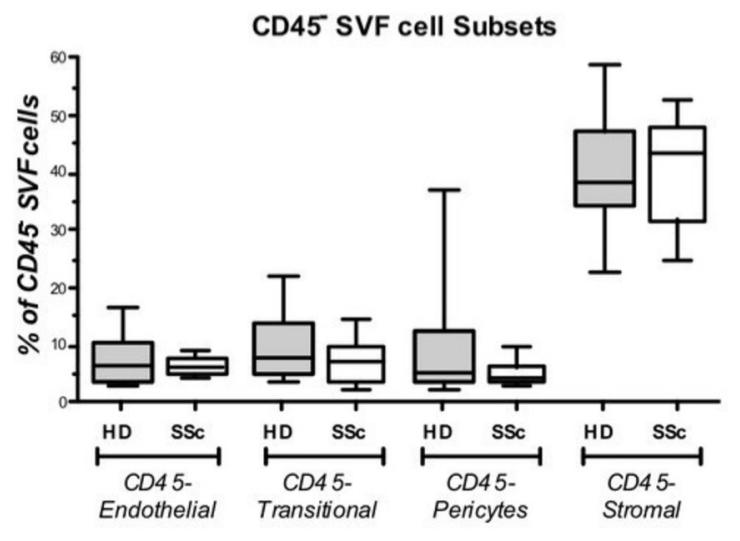

E

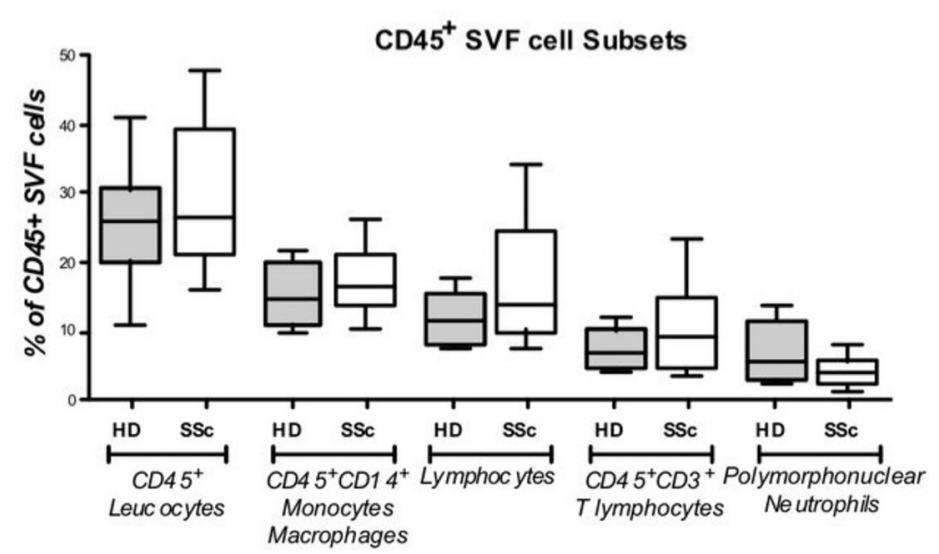

Figure 1 (A-C) The standard characteristics of SVF in HD and patients with SSC : viable nucleated cells yield, viability and clonogenic activity (CFU-F). (D) The repartition of CD45 - populations between HD and patients with SSC. (E) The repartition of CD45 + populations between HD and patients with SSc. CFU-F, colony-forming units-fibroblast; HD, healthy donors; SSc, systemic sclerosis.

Viable nucleated cells and colony-forming units-fibroblast

The standard characterisation of the SVF (Supplementary Figure) did not reveal any differences between patients with SSc and HD (figure $1 \mathrm{~A}, \mathrm{~B}, \mathrm{C}$ ) regarding the number of viable nucleated cells extracted/cc of fat (median 200469 [25-75th percentile range $123488-263167]$ in patients with SSc vs 214431 [180 $263-321616]$ in HD), cell viability (85\% [82-88] in SSc vs $84 \%$ [80-89] in HD) and the proportion of colony-forming units-fibroblast $(3.9 \%[2.3-5.4]$ in patients with SSc vs $2.7 \%$ [0.9-3.8] in HD).

\section{Flow cytometry analysis of SVF cell subsets}

The distribution of the cell subpopulations in SVF was not significantly different between patients with $\mathrm{SSc}$ and $\mathrm{HD}$ (figure 1D,E). The main cell subset presented an MSC phenotype (median: $43.9 \%$ [25-75 th percentile range: $31.8-48.5$ ] in patients with SSc vs $38.4 \%$ [34.0-47.3] in HD), whereas endothelial cells represented $6.1 \%$ of the cells (4.7-7.9) in patients with SSc vs $6.3 \%(3.3-10.5)$ in HD (figure 1D). The leucocytes (26.6\% [20.7-39.5] in patients with SSc vs 26\% [20.2-30.7] in HD) mainly consisted of lymphocyte and monocyte/macrophage populations (13.6\% [10.1-24.5] and 16.4\% [13.7-21.4] in patients with SSc vs $11.5 \%$ [7.9-15.7] and 15.0\% [11.520.3] in HD, respectively). In addition, a low proportion of polymorphonuclear cells was observed in both groups (4.3\% [2.6-5.8] in patients with SSc vs $5.5 \%$ [2.6-11.7] in HD) (figure 1E).

\section{Angiogenic activity of SVF cells}

The SVF-dependent formation of capillary-like structures, as evaluated in an in vitro Matrigel assay, was comparable in patients with SSc and HD (figure 2A). Sprout formation and the cumulative sprout length measured in a three-dimensional spheroid assay were not altered in SSc-SVF compared with HD-SVF (figure 2B, left graphs). However, a trend towards decreases in the average length of sprouts and the average number of junctions formed by sprouts was observed for SSc-SVF ( $p=0.07$ ) (figure $2 \mathrm{~B}$, right graphs). We then investigated the impact of SSc on SVF properties related to the formation of functional capillary networks in vivo using Matrigel plug assays performed in nu/nu mice. As controls, Matrigel implants that did not contain SVF were devoid of vessels (data not shown). H\&E histological staining revealed a significantly impaired capacity of SSc-SVF to generate vessels compared with HD-SVF (figure 2C, upper left graph). Furthermore, in the implants containing SSc-SVF, the number of Dextran+ perfused vessels was slightly decreased compared with the HD group, without reaching statistical significance $(p=0.24$; figure $2 \mathrm{C}$, upper right graph). Interestingly, this impairment of perfusion was also observed by ultrasound Doppler imaging, as indicated by a decrease in the vascularisation percentage $(p=0.11$; figure $2 \mathrm{C}$, lower right graph). Taken together, these data demonstrated that SVF from patients with SSc exhibited slightly impaired angiogenic potential. 
A

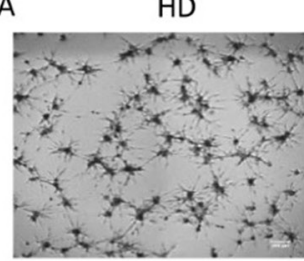

SSc
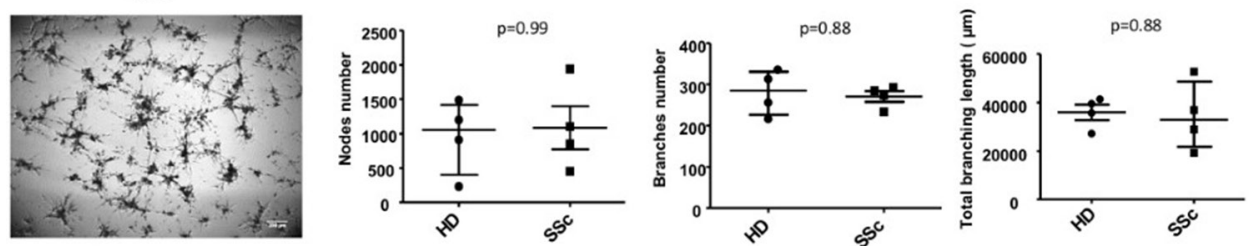

B
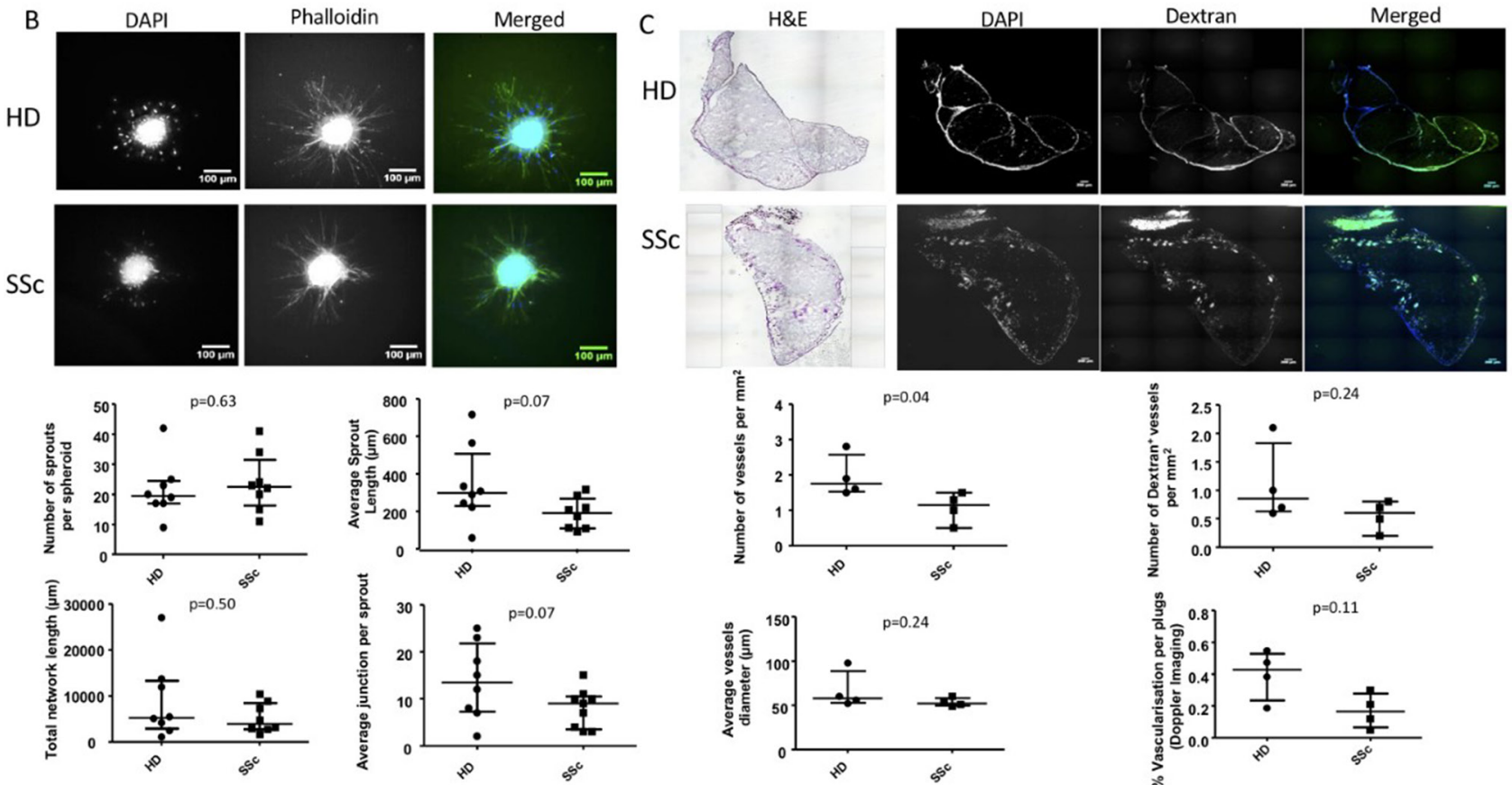

SSC
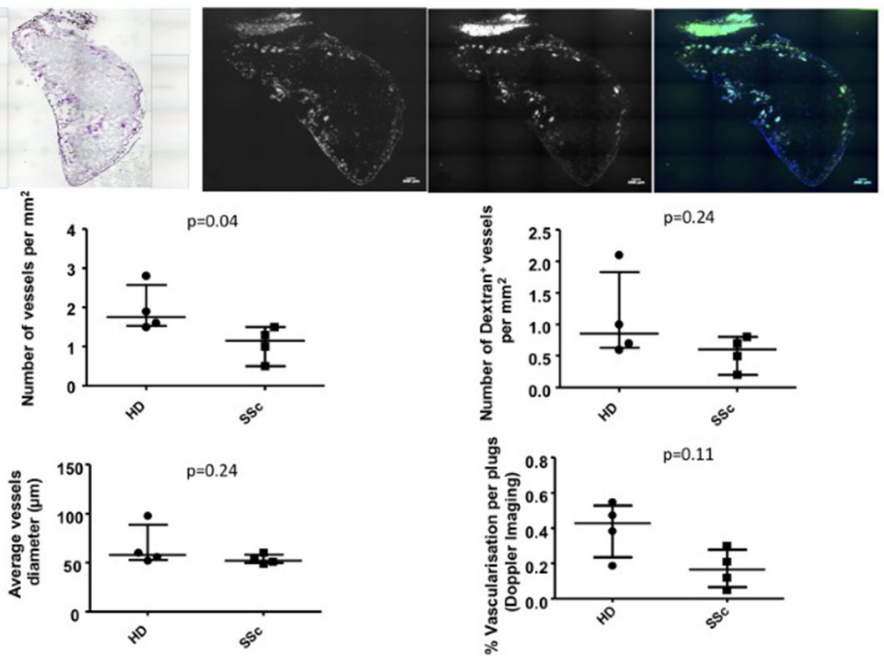

Figure 2 Angiogenic capacity of SVF cells from HD and patients with SSc. Matrigel tube formation did not reveal differences between groups (A). Spheroid-based sprouting assay revealed a tendency for higher angiogenic capacity of HD-SVF based on length and junction by sprouts (B). In vivo vascularisation revealed a significant increase in the number of vessels $/ \mathrm{mm}^{2}$ in $\mathrm{HD}$ conditions and a tendency for \% of vascularisation per plug using Doppler imaging (C). HD, healthy donors; SSC, systemic sclerosis; SVF, stromal vascular fraction.

\section{Transcriptomic analysis}

To explore the molecular mechanisms underlying the angiogenic potential of SSc-SVF, we performed global gene expression profiling. Hierarchical clustering distinguished a significant differential gene expression profile based on the comparison of patients with SSc and HD (figure 3A). Overall, the transcriptomic analysis revealed that 321 genes from the SVF samples were differentially expressed in patients with SSc compared with HD, among which 123 genes were upregulated and 198 were downregulated (online supplementary tables 2 and 3). Among these genes, six genes (ACE2, Apelin, IDO1, SAA1, GDF15 and HAS1) were selected for further confirmation of expression based on their previous implication in fibrosis or angiogenesis and the fold change in their differential expression between patients with SSc and HD. The quantitative reverse transcription-PCR analysis confirmed significant upregulation of the gene expression of ACE2, Apelin, IDO1 and GDF15 in SSc patients, while that of SAA1 and HAS1 only showed a statistical tendency towards upregulation and downregulation respectively (figure $3 \mathrm{~B}$ ). To provide a cohesive view of the biological functions associated with the changes in the SSc-SVF gene expression profile, we conducted a gene ontology analysis using the DAVID database. The upregulated genes showed a strong association with the positive regulation of cell proliferation as well as angiogenesis and vasculogenesis regulation, whereas the categories enriched among the downregulated genes were associated with the regulation of cell proliferation and migration, positive regulation of angiogenesis and the inflammatory response (figure 3C and online supplementary tables 4 and 5). Pathway enrichment analysis (online supplementary table 6) showed enrichment in several pathways involved in inflammatory response and angiogenesis (renin-angiotensin system, Notch), as well as pathways previously involved in SSc (transforming growth factor- $\beta$ [TGF$\beta]$ ). Collectively, these data identified an enrichment of genes involved in the control of vascular growth and maturation and the fibrotic response.

\section{Single-cell analysis}

A single-cell analysis was performed using four SVF samples (two HD, one limited and one diffuse cutaneous SSc samples). After normalisation, the gene content of 16590 cells was obtained ( 8619 for HD and 7971 for SSc) and 6 cell populations were distinguished based on gene expression levels. Identification of the different subpopulations was performed using the top 20 genes expressed by each population and the available literature on their specificity of expression. The CD45+ population comprised one myeloid population, representing 3.6\% of the analysed cells, and one lymphoid population (2.3\%). The CD45 - cells corresponded to stromal cells (46.8\%), pericytes $(27.8 \%)$ and two endothelial clusters $(15.8 \%$ and $3.8 \%$, respectively) (figure 4). Analysis of the set of genes of interest 


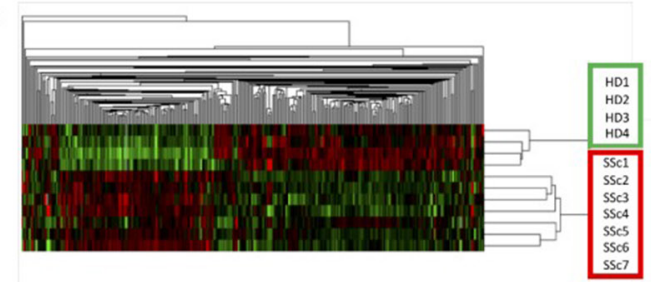

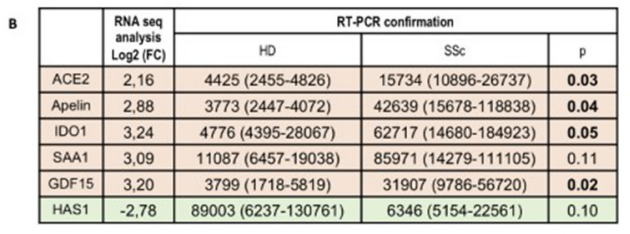

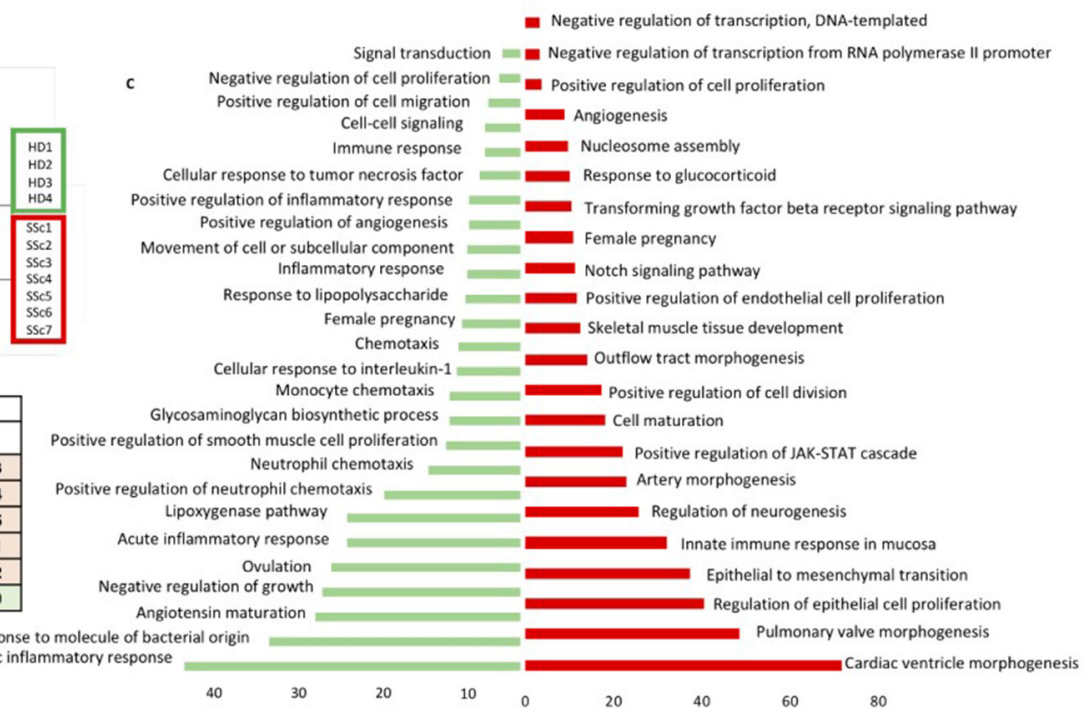

= Negative regulation of transcription, DNA-templated Figure 3 Transcriptomic analysis of SVF from HD ( $n=4)$ and patients with $S S C(n=7)$ revealed a differential expression profile between the two
conditions by hierarchic clustering (A) for 321 genes. (B) Represents the log2 (FC) obtained by RNA-Seq for selected genes and their confirmation by qRT-PCR (median [25\%-75\% quartile] expressed in specific transcripts detected per $10^{6}$ GAPDH transcripts). (C) Represents the fold enrichment over chance for the gene ontology biological process of the up (red) and down (green) gene lists using DAVID (fold change $\geq 2, p \leq 0.001$ ). FC, fold change; GADPH, glycéraldéhyde-3-phosphate déshydrogénase; $H D$, healthy donors; qRT-PCR, quantitative reverse transcription-PCR; SVF, stromal vascular fraction.

revealed major endothelial localisation of Apelin (71.7\%) and IDO1 (79.9\%), whereas SAA1 and GDF-15 were coexpressed by stromal cells ( $36.4 \%$ and $31.9 \%$, respectively) and one endothelial cluster (26.0\% and $58.0 \%$, respectively). The expression of HAS1 was specifically assigned to the stromal cell clusters, and ACE2 to pericytes. This analysis also confirmed the differential gene expression levels between patients with SSc and HD in specific subpopulations (online supplementary table 7).

\section{Secretome analysis}

Among the secreted proteins evaluated using Luminex assay, the SSc-SVF cells produced higher levels of SAA and GDF15, which was consistent with the transcript analysis. The SSc-SVF cells were also observed to secrete higher levels of VCAM1, ICAM1, ADAMTS 13 and P-selectin (figure 5). In addition, a trend towards increased levels of fibrinogen was observed in patients with SSc (online supplementary table 8).

\section{DISCUSSION}

In the context of regenerative medicine, SVF represents a promising therapy for ischaemic vasculopathy and hand disability in SSc. The use of an autologous source of SVF is advantageous to prevent alloimmune responses but assumes that the infused cell therapy product retains a favourable profile in terms of composition and functional properties, despite the disease context. Our study is the first to provide investigations on the autologous SVF used as an advanced therapeutic medical product in a phase II clinical trial addressing handicap of the hand in SSc. Importantly, we demonstrate that SSc does not compromise the vascular repair capacity of SVF and characterise the specific SSc-SVF molecular profile by RNA sequencing analysis.

The easily accessible and uncultured SVF, concentrating all regenerative cell components of the adipose tissue vasculature, was shown to promote the formation of robust self-assembled vasculature, making it a clinically relevant cellular system with therapeutic potential in vascular diseases. ${ }^{16}$ Biological reports, mainly investigating blood and bone marrow-derived cells, have documented that the SSc environment alters circulating or tissue-resident endogenous progenitor/stem cells in diverse ways. In addition, the mechanisms that sustain vasculopathy and fibrotic pathogenic processes associated with SSc involve a variety of cells, such as fibroblasts, pericytes, adipocytes, and endothelial cells and progenitors. However, the impact of SSc on the components of SVF has never been investigated. Based on the recent literature refining the phenotypic identification of SVF cell subsets, we designed a comprehensive flow cytometry approach, which showed no alteration in the distribution of the CD45 + leucocyte and CD45 - cell subsets in SVF derived from SSc adipose tissue. These results may indicate that quantitative SSc-associated microvascular alteration patterns, such as rarefaction of microvessels and perivascular immune cell infiltrates, are not significant in SVF. Of note, pericytes involved in fibrosis ${ }^{31}$ were also not altered in SSc-SVF. These observations attest that the homeostasis of SVF and the quantitative composition of the SVF-based cell therapy product are maintained in the autologous context of SSc.

Furthermore, in various complementary functional assays, we observed no major impairment in the angiogenic function of SSc-SVF. The only significant impact of SSc was shown to be a decrease in the density of the neovessels formed following the injection of SVF-loaded Matrigel in nude mice. These in vivo data are consistent with in vitro spheroid assays showing a slightly decreased sprouting capacity of SSc-SVF. Thus, we demonstrated that the vasculogenic behaviour of the SVF obtained from patients with SSc was only marginally affected, supporting the rationale for its therapeutic use. Previous studies in mice indicate that diabetes and age potentially alter the intrinsic ability of SVF to support microvascular network formation in vivo. ${ }^{32} 33$ Although the older age of the SSc cohort compared with HD might have contributed to the diminished neovascularisation potential of the SVF, this hypothesis is unlikely because a univariate analysis failed to associate age with any of the angiogenic features characterising SVF (data not shown). In addition, the heterogeneity of ongoing treatments among enrolled patients 


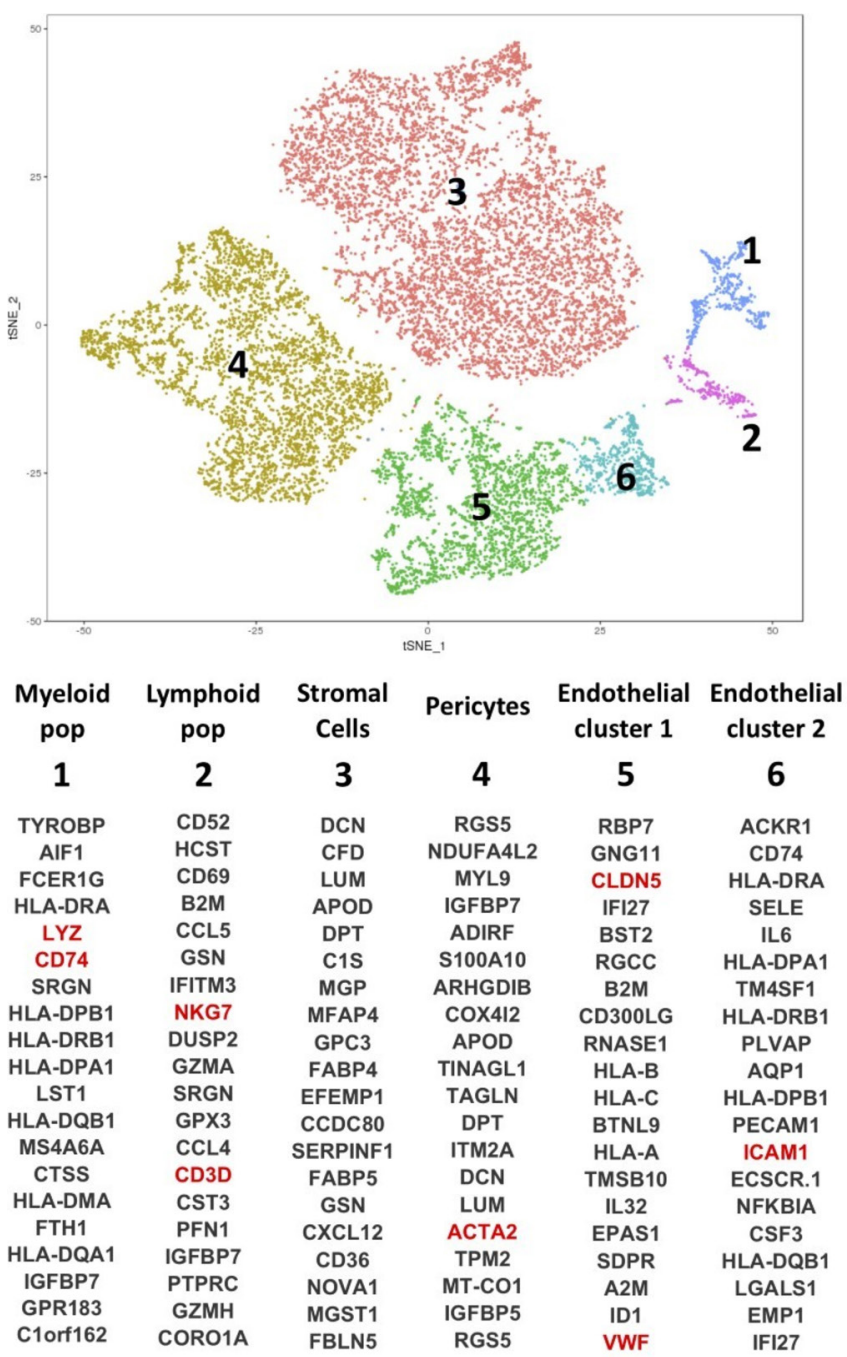

Figure 4 Graphic representation of single-cell analysis and top 20 genes expression for each cluster identified (in red, strong evidence genes). tSNE, t-distributed stochastic neighbor embedding.

with SSc did not allow delineating the specific impact of the pharmacological environment on these findings.

The current hypotheses about the angiogenic mechanism of action of SVF involve myeloid, immune cells ${ }^{34}$ and vascular wall resident stem/progenitor cells, ${ }^{3536}$ but highlight the major role of cooperation between MSC and EPC. The density of the vascular networks formed by the synergistic dual-cell system is many-fold higher than that found in implants containing either EPC or MSC alone. ${ }^{37}$ In such interplay, paracrine activity is known to make a predominant contribution. Factors produced by MSC, such as vascular endothelial growth factor (VEGF), aid in the migration and survival of EPC, whereas the platelet-derived growth factor (PDGF-BB) produced by EPC supports MSC proliferation and migration. ${ }^{37} 38$ Therefore, we further analysed whether SSc was associated with a specific molecular signature of SVF through global and single-cell RNA-Seq approaches and analysis of SVF-derived secreted factors. We provide the first evidence of a transcriptional signature with differential gene expression in whole SVF between SSc and healthy conditions. Notably, the transcript analysis identified a set of six genes of interest (Apelin, IDO1, SAA1, GDF15, HAS1 and ACE2) that are known to be involved in pathways controlling angiogenesis and fibrosis and are relevant to SSc vasculopathy. In this regard, the observed enrichment in several signalling pathways such as renin angiotensin system, TGF- $\beta$, Notch and PI3-kinase, is consistent with their involvement not only in SSc. These pathways also constitute key regulators of vascular stem/progenitor cells function. ${ }^{39}$ Interestingly, the expression of these genes was mainly assigned to clusters identified as cells belonging to the endothelial or stromal lineage using single-cell RNA-Seq technology.

Among the alterations predominantly affecting the endothelial SVF cell subset, we showed upregulation of Apelin gene expression in SSc-SVF. Apelin is an endothelial ligand of the Apelin receptor that activates proangiogenic signalling and regulates cardiovascular homeostasis. This result is in line with a previous description of enhanced levels of circulating Apelin as a marker of early endothelial activation and angiogenic responses that occur prior to fibrosis in early SSc and the development of proliferative vasculopathy in late-stage SSc. ${ }^{40} 41$ In addition, modulation of Apelin receptor was recently identified as a component of the SSc signature in skin endothelial cells analysed using single-cell RNA sequencing approach. ${ }^{42}$ We also bring a first evidence of an increase in endothelial indoleamine 2,3-dioxygenase 1 (IDO1) expression in SSc-SVF. IDO1 catalyses the production of metabolites in the Kynurenine pathway of tryptophan degradation. In addition to its well-known immunoregulatory functions, IDO1 was shown to be involved in the regulation of endothelial function, vascular tone, angiogenesis and atherogenesis processes. ${ }^{43}$ Consistent with our results, IDO is emerging as a key regulator of innate and adaptive immune inflammatory responses in SSc. ${ }^{44}$ Thus, upregulation of IDO in SVF could be a compensatory mechanism that counteracts endothelial inflammation, dysangiogenesis and immune activation. Moreover, an analysis of conditioned media from SSc-SVF revealed higher levels of the endothelial activation markers VCAM1, P-selectin and ICAM1 compared with HD-SVF. Collectively, these data support the hypothesis of an activated endothelial profile induced by SSc in adipose tissue. These also suggest that Apelin and IDO1 could be targeted in an attempt to improve SVF therapeutic potential.

In addition to these endothelial-specific alterations, the enhanced GDF15 and SAA1 secretion and gene expression observed in SSc-SVF were localised to both stromal and endothelial cells. GDF15 is involved in fibrosis initiation in scleroderma. ${ }^{45-47}$ Serum levels of SAA1 are higher in patients with SSc with pulmonary involvement ${ }^{48}$ and in patients presenting giant cell arteritis compared with HDs with potential myofibroblast outgrowth induction. ${ }^{49}$ In addition, HAS1 transcripts were significantly downregulated in SSc-SVF, and their expression was mainly assigned to stromal cells based on single-cell analysis. HAS1 plays a role in synthesising hyaluronic acid. ${ }^{50}$ Collectively, the significance of such alterations deserves further investigation, as they may affect the impact of SSc-SVF on fibrosis.

In conclusion, our study is the first to show that the quantitative distribution of the endothelial, stromal, immune and pericytes cell subsets is preserved within the autologous SVF preparation used to treat patients with SSc. While the transcriptomic and secretory profiles of SVF obtained from patients with SSc exhibited a molecular signature that reflected fibrosis, angiogenesis and endothelial activation processes, we provide functional evidence that these alterations did not translate into major impairment of the angiogenic behaviour of the autologous SVF medicinal product. However, specific profiles that may account for the interindividual variability in SSc-SVF potency could impact the cell-based therapeutic effect. Taking advantage of the ongoing clinical trial assessing the fully characterised autologous SVF batches used to treat hand disability in patients with SSc, a future analysis will allow us to determine the relationship 


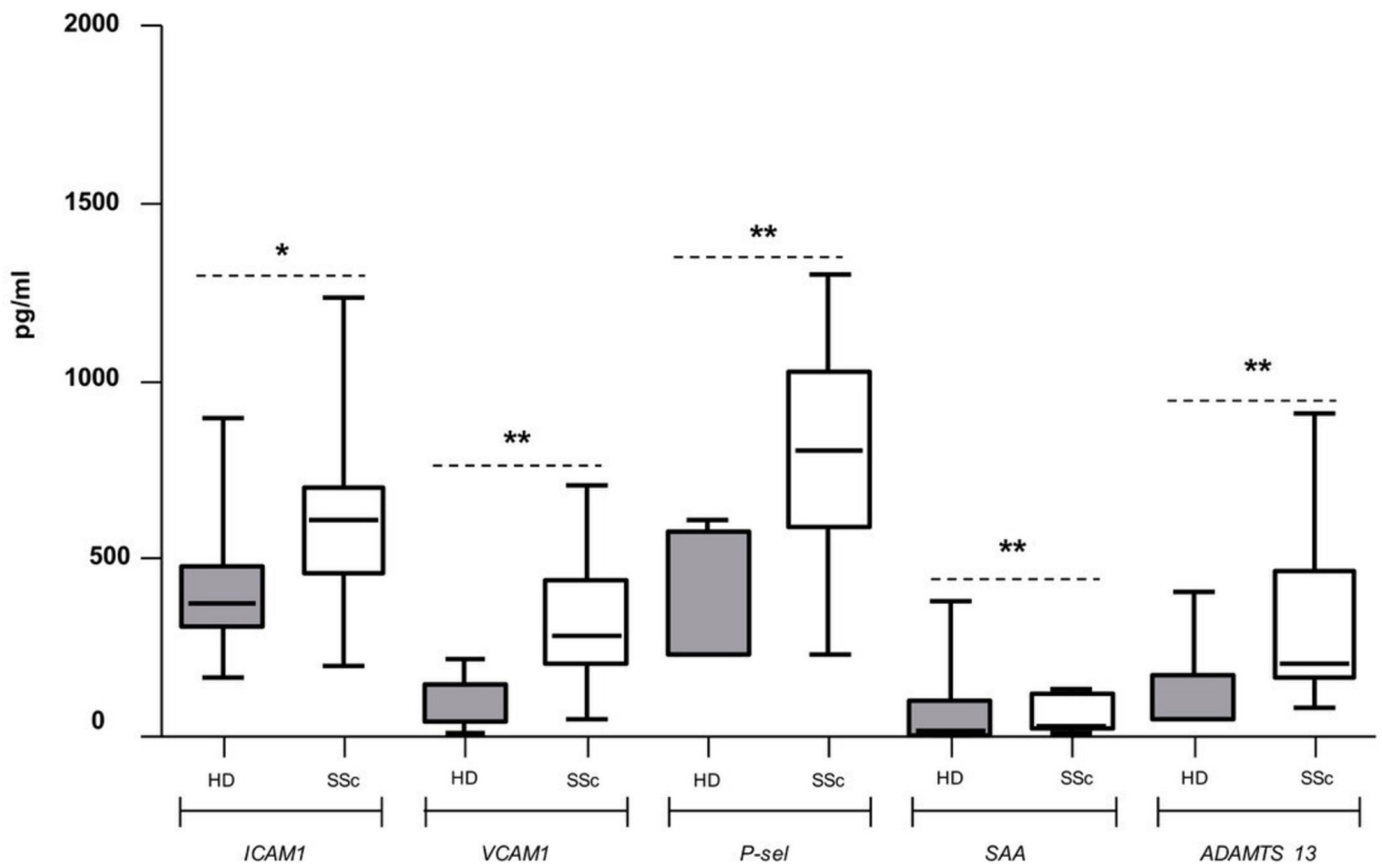

Figure 5 Significant differences $(p<0.05)$ in secreted proteins from the stromal vascular fraction of patients with systemic sclerosis (SSc) and healthy donors (HD).

between the biological attributes of SVF and its clinical efficacy in reducing vascular manifestations, which should provide clues for identifying markers of a good responder profile. These results also provide new perspectives and targets for delineating potential cell engineering strategies to optimise SVF-based therapeutic approaches in a personalised way.

\section{Author affiliations \\ ${ }^{1}$ Cell Therapy Department, Hôpital de la Conception, AP-HM, INSERM CIC BT 1409, Marseille, France \\ ${ }^{2}$ INSERM, INRA, C2VN, Aix-Marseille University, Marseille, France \\ ${ }^{3}$ Plastic Surgery Department, Hôpital de la Conception, AP-HM, Marseille, France \\ ${ }^{4}$ Internal Medicine Department, Hôpital Nord \& Hôpital de la Timone, AP-HM, Marseille, France \\ ${ }^{5}$ Urology Surgery Department, Hôpital de la Conception, AP-HM, Marseille, France \\ ${ }^{6}$ Vascular Biology Department, Hôpital de la Conception, AP-HM, Marseille, France ${ }^{7}$ CERIMED, Aix-Marseille University, AP-HM, Marseille, France}

Contributors Study concept and design: JM, SS, PP, FS. Collection and acquisition of data: JM, MV, BB, AD, AB, RB. Analysis and interpretation of data: JM, PF, LA, LL. In vivo experiments including interpretation: $L L, S F, B e G$. Drafting of the manuscript: $J M, P F, M V, B B$. Critical revision of the manuscript for important intellectual content: FD-G, BeG, BrG, PP, FS . Study supervision: FD-G, DC, PP, FS.

Funding Funding for this work was granted by Fondation de l'Avenir (Grant APM RMA 2015-0008) and AORC Junior 2017 from Assistance Publique Hopitaux Marseille.

Disclaimer We certify that the manuscript was edited for proper English language, grammar, punctuation, spelling and overall style by one or more of the highly qualified native English-speaking editors at American Journal Experts (Certificate Verification Key 9067-71A5-5CFD-7DC1-18AP).

Competing interests None declared.

Patient consent for publication Obtained.

Ethics approval The protocol was approved by the local ethics committee (authorisation \#14.051 from CPP Sud Méditerranée V) and the National Health Regulatory Authority (authorisation \#141 449 A-62 from ANSM).

Provenance and peer review Not commissioned; externally peer reviewed.

\section{REFERENCES}

1 Denton CP, Khanna D. Systemic sclerosis. Lancet 2017;390:1685-99.
2 Allanore Y, Distler O, Matucci-Cerinic M, et al. Review: defining a unified vascular phenotype in systemic sclerosis. Arthritis Rheumatol 2018;70:162-70.

3 Nikpour M, Baron M. Mortality in systemic sclerosis: lessons learned from populationbased and observational cohort studies. Curr Opin Rheumatol 2014;26:131-7.

4 Tyndall AJ, Bannert B, Vonk M, et al. Causes and risk factors for death in systemic sclerosis: a study from the EULAR Scleroderma Trials and Research (EUSTAR) database. Ann Rheum Dis 2010;69:1809-15.

5 Poudel DR, Jayakumar D, Danve A, et al. Determinants of mortality in systemic sclerosis: a focused review. Rheumatol Int 2018:38:1847-1858:1847-58.

6 Young A, Namas R, Dodge C, et al. Hand impairment in systemic sclerosis: various manifestations and currently available treatment. Curr Treatm Opt Rheumatol 2016;2:252-69.

7 Araújo FC, Camargo CZ, Kayser C. Validation of the ACR/EULAR classification criteria for systemic sclerosis in patients with early scleroderma. Rheumatol Int 2017:37:1825-33.

8 Sullivan KM, Goldmuntz EA, Keyes-Elstein L, et al. Myeloablative autologous stem-cell transplantation for severe scleroderma. N Engl J Med 2018;378:35-47.

9 Burt RK, Farge D. Systemic sclerosis: autologous HSCT is efficacious, but can we make it safer? Nat Rev Rheumatol 2018;14:189-91.

10 Kowal-Bielecka O, Fransen J, Avouac J, et al. Update of EULAR recommendations for the treatment of systemic sclerosis. Ann Rheum Dis 2017;76:1327-39.

11 Maria AT, Maumus M, Le Quellec A, et al. Adipose-derived mesenchymal stem cells in autoimmune disorders: state of the art and perspectives for systemic sclerosis. Clin Rev Allergy Immunol 2017;52:234-59.

12 Maria AT, Toupet K, Maumus M, et al. Human adipose mesenchymal stem cells as potent anti-fibrosis therapy for systemic sclerosis. J Autoimmun 2016:70:31-9.

13 Maria AT, Toupet K, Bony C, et al. Antifibrotic, antioxidant, and immunomodulatory effects of mesenchymal stem cells in $\mathrm{HOCl}$-Induced systemic sclerosis. Arthritis Rheumatol 2016;68:1013-25.

14 Scuderi N, Ceccarelli S, Onesti MG, et al. Human adipose-derived stromal cells for cell-based therapies in the treatment of systemic sclerosis. Cell Transplant 2013;22:779-95

15 Bora P, Majumdar AS. Adipose tissue-derived stromal vascular fraction in regenerative medicine: a brief review on biology and translation. Stem Cell Res Ther 2017;8:145.

16 Ramakrishnan VM, Boyd NL. The adipose stromal vascular fraction as a complex cellular source for tissue engineering applications. Tissue Eng Part B Rev 2018:24:289-99.

17 Klar AS, Güven S, Zimoch J, et al. Characterization of vasculogenic potential of human adipose-derived endothelial cells in a three-dimensional vascularized skin substitute. Pediatr Surg Int 2016;32:17-27.

18 Poitevin S, Cussac D, Leroyer AS, et al. Sphingosine kinase 1 expressed by endothelial colony-forming cells has a critical role in their revascularization activity. Cardiovasc Res 2014;103:121-30. 
19 Granel B, Daumas A, Jouve E, et al. Safety, tolerability and potential efficacy of injection of autologous adipose-derived stromal vascular fraction in the fingers of patients with systemic sclerosis: an open-label phase I trial. Ann Rheum Dis 2015;74:2175-82.

20 Daumas A, Magalon J, Jouve E, et al. Long-term follow-up after autologous adiposederived stromal vascular fraction injection into fingers in systemic sclerosis patients. Curr Res Trans/ Med 2017;65:40-3.

21 Koh YJ, Koh BI, Kim H, et al. Stromal vascular fraction from adipose tissue forms profound vascular network through the dynamic reassembly of blood endothelial cells. Arterioscler Thromb Vasc Biol 2011;31:1141-50.

22 Serratrice N, Bruzzese L, Magalon J, et al. New fat-derived products for treating skininduced lesions of scleroderma in nude mice. Stem Cell Res Ther 2014;5:138.

23 Comella K, Parcero J, Bansal H, et al. Effects of the intramyocardial implantation of stromal vascular fraction in patients with chronic ischemic cardiomyopathy. J Trans/ Med 2016:14:158.

24 Mostmans Y, Cutolo M, Giddelo C, et al. The role of endothelial cells in the vasculopathy of systemic sclerosis: A systematic review. Autoimmun Rev 2017;16:774-86.

25 Brunasso AM, Massone C. Update on the pathogenesis of Scleroderma: focus on circulating progenitor cells. F1000Res 2016;5:723.

26 Vanneaux V, Farge-Bancel D, Lecourt S, et al. Expression of transforming growth factor $\beta$ receptor II in mesenchymal stem cells from systemic sclerosis patients. BMJ Open 2013;3:e001890.

27 Cipriani P, Marrelli A, Benedetto PD, et al. Scleroderma mesenchymal stem cells display a different phenotype from healthy controls; implications for regenerative medicine. Angiogenesis 2013;16:595-607.

28 Capelli C, Zaccara E, Cipriani P, et al. Phenotypical and functional characteristics of in vitro-expanded adipose-derived mesenchymal stromal cells from patients with systematic sclerosis. Cell Transplant 2017;26:841-54

29 Manetti M, Romano E, Rosa I, et al. Endothelial-to-mesenchymal transition contributes to endothelial dysfunction and dermal fibrosis in systemic sclerosis. Ann Rheum Dis 2017;76:924-34.

30 Avouac J, Cagnard N, Distler JH, et al. Insights into the pathogenesis of systemic sclerosis based on the gene expression profile of progenitor-derived endothelial cells. Arthritis Rheum 2011;63:3552-62.

31 Rajkumar VS, Howell K, Csiszar K, et al. Shared expression of phenotypic markers in systemic sclerosis indicates a convergence of pericytes and fibroblasts to a myofibroblast lineage in fibrosis. Arthritis Res Ther 2005;7:R1113-23.

32 Cianfarani F, Toietta G, Di Rocco G, et al. Diabetes impairs adipose tissue-derived stem cell function and efficiency in promoting wound healing. Wound Repair Regen 2013;21:545-53.

33 Aird AL, Nevitt CD, Christian K, et al. Adipose-derived stromal vascular fraction cells isolated from old animals exhibit reduced capacity to support the formation of microvascular networks. Exp Gerontol 2015;63:18-26.

34 Navarro A, Marín S, Riol N, et al. Human adipose tissue-resident monocytes exhibit an endothelial-like phenotype and display angiogenic properties. Stem Cell Res Ther 2014;5:50.
35 Awgulewitsch CP, Trinh LT, Hatzopoulos AK. The vascular wall: a plastic hub of activity in cardiovascular homeostasis and disease. Curr Cardiol Rep 2017;19:51.

36 Wörsdörfer P, Mekala SR, Bauer J, et al. The vascular adventitia: An endogenous, omnipresent source of stem cells in the body. Pharmacol Ther 2017;171:13-29.

37 Traktuev DO, Prater DN, Merfeld-Clauss S, et al. Robust functional vascular network formation in vivo by cooperation of adipose progenitor and endothelial cells. Circ Res 2009:104:1410-20.

38 Traktuev DO, Merfeld-Clauss S, Li J, et al. A population of multipotent CD34positive adipose stromal cells share pericyte and mesenchymal surface markers, reside in a periendothelial location, and stabilize endothelial networks. Circ Res 2008; 102:77-85.

39 Lu W, Li X. Vascular stem/progenitor cells: functions and signaling pathways. Cell Mol Life Sci 2018;75:859-69.

40 Wiwanitkit V. Serum apelin levels in systemic sclerosis. J Eur Acad Dermato/ Venereol 2013;27:1586

41 Aozasa N, Asano Y, Akamata K, et al. Serum apelin levels: clinical association with vascular involvements in patients with systemic sclerosis. J Eur Acad Dermatol Venereol 2013;27:37-42.

42 Apostolidis SA, Stifano G, Tabib T, et al. Single Cell RNA Sequencing Identifies HSPG2 and APLNR as markers of endothelial cell injury in systemic sclerosis skin. Front Immunol 2018:9:2191.

43 Song P, Ramprasath T, Wang H, et al. Abnormal kynurenine pathway of tryptophan catabolism in cardiovascular diseases. Cell Mol Life Sci 2017;74:2899-916

44 Legány N, Berta L, Kovács L, et al. The role of B7 family costimulatory molecules and indoleamine 2,3-dioxygenase in primary Sjögren's syndrome and systemic sclerosis. Immunol Res 2017:65:622-9.

45 Lambrecht S, Smith V, De Wilde K, et al. Growth differentiation factor 15, a marker of lung involvement in systemic sclerosis, is involved in fibrosis development but is not indispensable for fibrosis development. Arthritis Rheumatol 2014;66:418-27.

46 Gamal SM, Elgengehy FT, Kamal A, et al. Growth Differentiation Factor-15 (GDF15) level and relation to clinical manifestations in egyptian systemic sclerosis patients: preliminary data. Immunol Invest 2017:46:703-13.

47 Yanaba K, Asano Y, Tada Y, et al. Clinical significance of serum growth differentiation factor-15 levels in systemic sclerosis: association with disease severity. Mod Rheumatol 2012;22:668-75.

48 Lakota K, Carns M, Podlusky S, et al. Serum amyloid A is a marker for pulmonary involvement in systemic sclerosis. PLoS One 2015;10:e0110820.

49 O'Neill L, Rooney P, Molloy D, et al. Regulation of inflammation and angiogenesis in giant cell arteritis by acute-phase serum Amyloid A. Arthritis Rheumatol 2015;67:2447-56.

50 Chan DD, Xiao WF, Li J, et al. Deficiency of hyaluronan synthase 1 (Has 1) results in chronic joint inflammation and widespread intra-articular fibrosis in a murine model of knee joint cartilage damage. Osteoarthritis Cartilage 2015;23:1879-89 